\title{
Endgroup Determination of Synthetic Polymers by Electrospray Ionization Fourier Transform Ion Cyclotron Resonance Mass Spectrometry
}

\author{
Sander Koster, Marc C. Duursma, Jaap J. Boon, and Ron M. A. Heeren \\ Unit for Macromolecular Mass Spectrometry, FOM-Institute for Atomic and Molecular Physics, Amsterdam, \\ The Netherlands
}

\begin{abstract}
Electrospray ionization (ESI) was performed on a Fourier transform ion cyclotron resonance mass spectrometer for the endgroup and monomer mass determination of three poly(oxyalkylene)s in the mass range of 400-8000 Da. A combined use of the multiple charge states observed with ESI, leads to a threefold increase in accuracy of the endgroup and monomer determination. The improvement is attributed to the increased number of datapoints used for the regression procedure, yielding more accurate results. Endgroup masses are determined with a mass error better than 5 and 75 millimass units for the molecular weight range of 400-4200 and 6200-8000 Da, respectively. A mass error of better than 1 millimass unit was observed for all monomer mass determinations. With ESI, endgroup and monomer masses have been determined for poly(ethylene glycol) oligomers with a mass higher than $8000 \mathrm{Da}$. This is almost two times higher than observed with matrix-assisted laser desorption/ ionization on the same instrument. (J Am Soc Mass Spectrom 2000, 11, 536-543) (C) 2000 American Society for Mass Spectrometry
\end{abstract}

S ynthetic polymers consist of a distribution of oligomers because of the statistical nature of the polymerization process. Various physical, mechanical, and rheological properties are related to the width of the molecular weight distribution and the composition of the monomers and endgroup. Therefore, an improvement in the characterization techniques for synthetic polymers is of high importance. In this paper we will focus on an improvement of the determination of the monomer and endgroup mass/structure using mass spectrometry.

One of the most commonly used techniques to obtain endgroup information is endgroup titration. However, this technique fails to provide information about the structure of the monomer. NMR can provide information on both the endgroup and monomer structure but only yields an average of these polymer characteristics. Mass spectrometry can provide detailed molecular information of composition and structure for a large variety of polymer classes using only minute amounts of sample. Matrix-assisted laser desorption/ionization time of flight mass spectrometry (MALDI TOF-MS) has proven to be a good technique for the characterization of the molecular weight distribution and the monomer/ endgroup composition up to a molecular weight of $\sim 50,000 \mathrm{Da}$ [1-3]. However, for high $\mathrm{m} / z$ the resolution

Address reprint requests to Dr. R. M. A. Heeren, Kruislaan 407, 1098SJ Amsterdam, The Netherlands. E-mail: heeren@amolf.nl is not sufficient to distinguish the different isotopic peaks. Isotopic resolution is not necessary for the determination of the molecular weight distribution but for an accurate endgroup and monomer mass determination isotopic resolution is required as will be shown in this paper.

Fourier transform ion cyclotron resonance mass spectrometry (FTICR-MS) has many advantages over conventional mass spectrometric techniques. For polymer studies most relevant advantages are high mass resolving power and high mass accuracy. Masses of $\mathrm{m} / \mathrm{z}$ $\sim 4000$ can be measured with a mass accuracy of 1-10 ppm in broadband mode [4-6]. Measurements carried out in high resolution mode provide resolutions $>10^{6}$ and a mass accuracy of $<1 \mathrm{ppm}$ [7]. As has been shown, these features can be exploited for the characterization of synthetic polymers with MALDI FTICR-MS [6]. However, given the single charge state observed with MALDI, the technique is limited to relatively lowmolecular weight polymeric systems because high mass ions (high $\mathrm{m} / \mathrm{z}$ ) are more difficult to trap [8,9]. A technique that overcomes this problem is electrospray ionization (ESI). The main advantage of ESI compared to other ionization techniques is that multiply charged ions of the polymer of interest are formed enabling analysis at lower $\mathrm{m} / \mathrm{z}$ values. ESI also provides a low internal energy deposition resulting in minimal fragmentation of the polymer of interest. ESI can be used online with SEC, spraying conventional solutions [10, 
11]. Solubility properties and the inability to interact with cations can be a limitation for polymer analysis (e.g., polyolefins) [12].

Because of the multiple charge states created by ESI, overlapping distributions of polymeric ions are observed in the mass spectra. Mass spectrometers such as quadrupoles [10,13,14], sector, and TOF instruments [15] are unable to distinguish the different charge states due to their relatively low resolution. The combination of ESI and FTICR-MS provides enough resolution to distinguish the isotopic peaks of the oligomers in the different charge states of a molecular weight up to 23,000 [18]. ESI FTICR-MS has mainly been used for the characterization of peptides and proteins $[16,17]$. Only a few research groups have used ESI FTICR-MS for the characterization of synthetic polymers [18, 19], whereas most groups use MALDI [4, 8, 9, 20-24].

We have combined the advantages of ESI and FTICR-MS for the characterization of poly(ethylene glycol) (PEG), poly(propylene glycol) (PPG), and poly(tetrahydrofuran) (PTHF) on monomer and endgroup mass. A novel method is introduced that uses all charge states in one linear regression procedure. We will demonstrate how this method can be used to improve the endgroup characterization substantially compared to the linear regression procedure using a single charge state, as done with MALDI.

\section{Experimental}

ESI FTICR-MS experiments are performed on a modified Bruker-Spectrospin (Fällanden, Switzerland) APEX 7.0e FTICR-MS equipped with a 7 tesla superconducting magnet. The ICR cell is an Infinity Cell [25]. The system, except for the ESI source, has been described earlier $[26,27]$. The pressure in the ICR cell is held at $10^{-9}-10^{-10}$ mbar. Five stages of differential pumping are employed to bridge the atmospheric pressure conditions in the ESI source. The sample is pumped with a Harvard syringe pump model 55-1111 (Kent, U.K.) at a flowrate of $0.1 \mathrm{~mL} / \mathrm{h}$ through a fused silica capillary ( $0.18 \mathrm{~mm}$ internal diameter). Positively charged electrosprayed droplets generated by applying a $\pm 3000 \mathrm{~V}$ potential to a stainless steel spray needle are accelerated towards a stainless steel heater capillary at $100 \mathrm{~V}$. The sharpened $\left(\sim 45^{\circ}\right)$ spray needle has an internal diameter of $0.25 \mathrm{~mm}$ and is positioned $\sim 0.5 \mathrm{~cm}$ from the heater capillary. The heater capillary has an internal diameter of $0.75 \mathrm{~mm}$, length of $20 \mathrm{~cm}$ (Alltech Assoc., Inc., Deerfield, IL) and is placed in a ceramic heater tube. To aid the evaporation of the solvent, the stainless steel capillary is held at approximately $170{ }^{\circ} \mathrm{C}$ by passing a current of $1.8 \mathrm{~A}(\sim 12 \mathrm{~W})$ through the ceramic heater tube and the capillary.

In the first pumping stage, the nozzle skimmer region, the pressure is approximately $1 \mathrm{mbar}$. The ions are focused by a tube lens through a copper skimmer with an orifice diameter of $1 \mathrm{~mm}$. The skimmer removes the excess neutrals. Typical nozzle skimmer voltage differences are about $5 \mathrm{~V}$. The second pumping stage contains a rf-only quadrupole at a pressure of $10^{-5}$ mbar. At the exit of the quadrupole, ions are accelerated to $3000 \mathrm{eV}$ to prevent radial ejection by the magnetic field. Electrostatic ion optics are used (at a pressure of $10^{-7}$ mbar) to guide the ions from the exit of the quadrupole to the ICR cell. Before entering the ICR cell the ions are decelerated to approximately $1 \mathrm{eV}$ to facilitate trapping in the ICR cell at $10^{-9}-10^{-10}$ mbar. Data (128k datapoints) acquisition and control is performed using XMASS (Bruker-Daltonics, Billerica, MA).

The poly(ethylene glycol) (PEG): PEG3000 and PEG6000 and poly(propylene glycol) (PPG): PPG1150 and PPG3250 standards are obtained from Fluka Chemical (Buchs, Switzerland). PEG1000 is obtained from Serva (Heidelberg, Germany). The poly(tetra hydrofuran) PTHF2000 sample is supplied by DSM (Geleen, The Netherlands). The numbers in the names denote the average molecular weight of the sample. All samples contain sodium from origin and are used without further purification. The PEG samples are sprayed in an $\sim 1 \mu \mathrm{M}$ solution with a solvent composition of 70:30 $\mathrm{MeOH}: \mathrm{H}_{2} \mathrm{O}$. PPG and PTHF are sprayed with concentrations of $\sim 5$ and $100 \mu \mathrm{M}$, respectively. The average molecular weight of the polymer standards (e.g., 3000 $\mathrm{g} / \mathrm{mol}$ for PEG3000) is used for the calculation of the polymer concentration. Approximately $10 \mu \mathrm{M} \mathrm{NaI}$ (Aldrich) was added to the solutions to be sprayed to increase the $\mathrm{S} / \mathrm{N}$ ratio.

\section{Results and Discussion}

A series of poly(oxyalkylene)s with different average molecular weights in the range 400-8000 Da was used for endgroup determination. Charge states of $1+$ to $12+$ are observed depending on the average molecular weight of the polymer standards. In Figure 1 the spectra of PEG3000, PPG3250, and PTHF2000 are shown. All oligomer peaks are isotopically resolved (see Figure 1) with a resolution $(\mathrm{m} / \Delta \mathrm{m})_{50 \%}$ of $\sim 25,000$ at $\mathrm{m} / \mathrm{z} \sim 1000$ in broadband mode. Low charge states are predominantly observed on small oligomers, whereas the higher charge states are mainly found for the larger oligomers.

The spectrum of PEG6000 is more complex than the spectra of PEG1000 and PEG3000 even though the samples contain approximately an equal numbers of oligomers. This difference is caused by three factors. First, higher mass oligomers are observed in more charge state distributions. Second, the spacing between the oligomer and isotopic peaks becomes smaller for higher charge states. Third, the number of isotopic peaks increases with the degree of polymerization. Figure 2 shows a simulation of the isotopic distribution of three oligomers (upper figures) with degrees of polymerization 25,75 , and 150 which are comparable to an average oligomer in PEG1000, PEG3000, and PEG6000, respectively. Four isotopic peaks are observed for PEG1000, whereas for a PEG6000 oligomer about 10 isotopic peaks show up in the spectrum. 


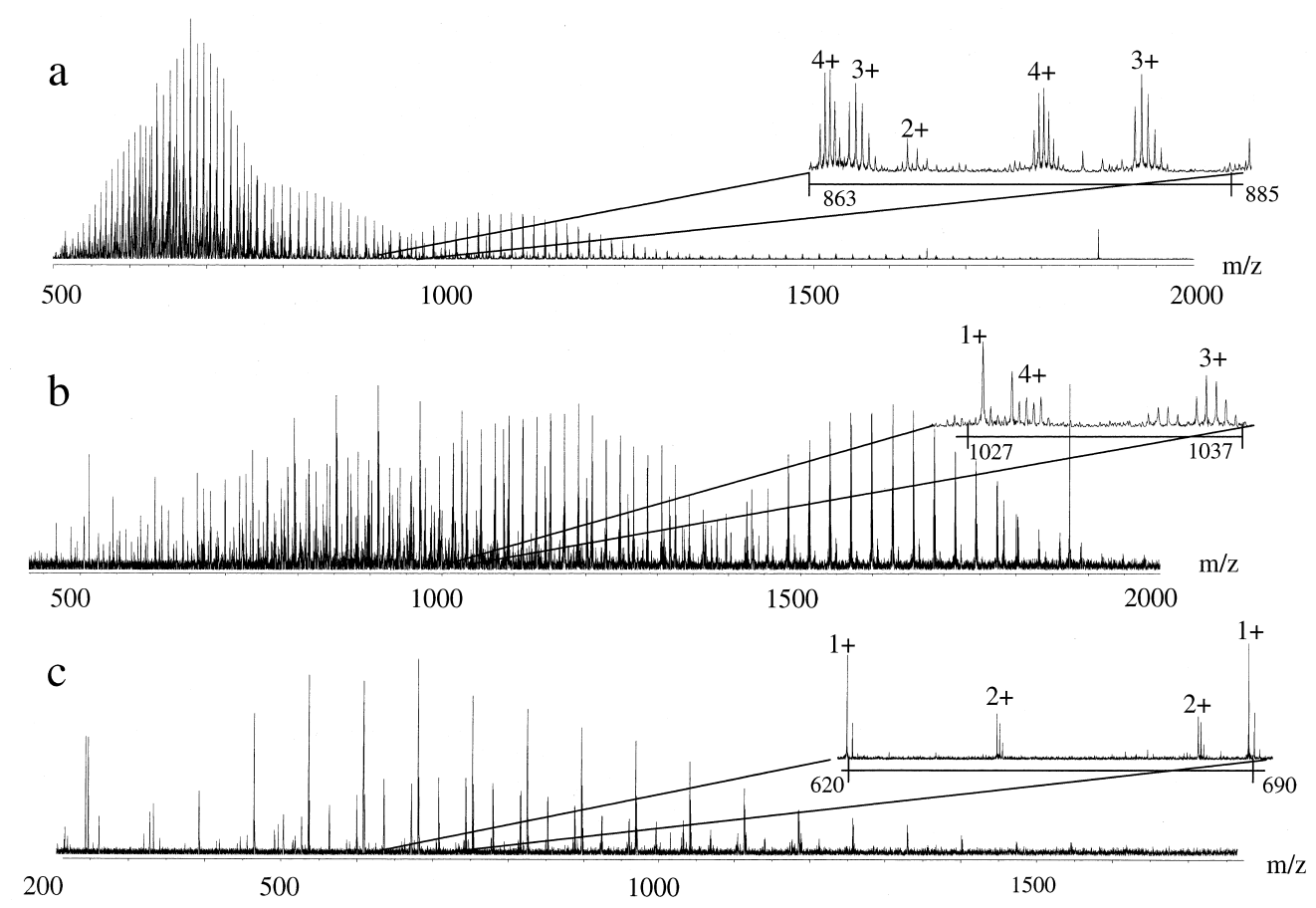

Figure 1. ESI FTICR-MS spectra of (a) PEG3000, (b) PPG3250, and (c) PTHF2000 with sodium iodide added to the spray solution (70:30 $\left.\mathrm{MeOH}: \mathrm{H}_{2} \mathrm{O}\right)$.

Another feature in Figure 2 is that the intensity of the mono isotopic peak decreases with increasing molecular weight; for PEG6000, the intensity has become almost zero. This is because the probability to observe a molecule containing only ${ }^{12} \mathrm{C}$ atoms decreases with an increasing number of carbon atoms. Some of the experimentally observed isotopic peaks of PEG6000 (lower figure) show small deviations from the simulated isotopic pattern. This is probably caused by an overlap with peaks of other oligomers. In general, the experimentally observed isotopic patterns correspond well with the simulated pattern.
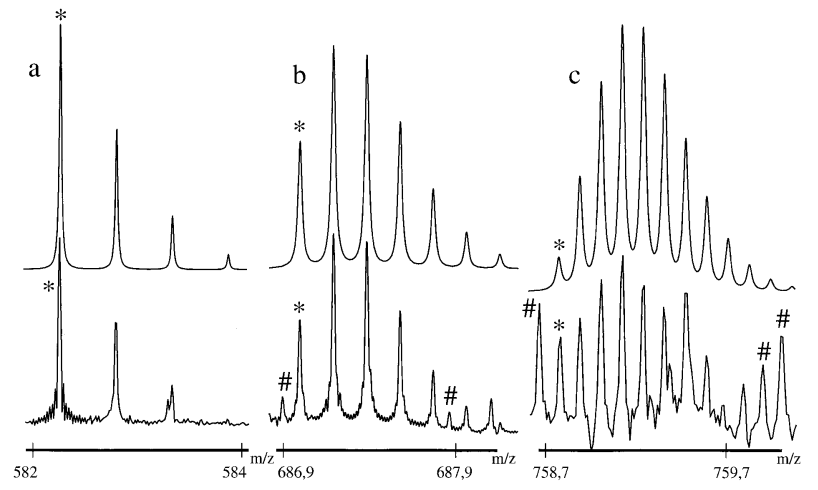

Figure 2. Simulation (upper) and experimentally observed isotopic pattern of PEGs with (a) $n=25, z=2$, (b) $n=75, z=5$, and (c) $n=150, z=9$. Peaks marked with an asterisk are the mono isotopic peaks. Peaks marked with a mesh sign originate from other oligomers.
Theory of the Endgroup Determination Using the Linear Regression and Averaging Methods with Electrospray Ionization

Two methods used for the calculation of the endgroup mass, monomer mass, and mass accuracy applied to MALDI FTICR-MS have been described in a previous article [6]. The multiple charge states observed with ESI require a linear regression procedure similar to that applied to the MALDI data in which only singly charged ions are considered. In ESI, an ion in charge state $z$ will contain $z$ cations. The charge state is determined from the $\mathrm{m} / \mathrm{z}$ spacing between the carbon isotopic peaks. Because $z$ can be very large, and the mass accuracy of the FTICR-MS is very high, the mass of $z$ electrons is taken into account. Fenn et al. estimated more than 4200 charges on a PEG with a molecular weight of 5 million [28]. The measured mass over charge ratio, $(\mathrm{m} / \mathrm{z})_{\text {meas }}^{z+}$, for the monoisotopic oligomer is given by

$$
\begin{aligned}
\left(\frac{m}{z}\right)_{\text {meas }}^{z+} & =\frac{n \cdot m_{\text {mon }}+m_{\mathrm{end}}+z \cdot m_{\mathrm{cat}}-z \cdot m_{\mathrm{elec}}}{z} \\
& =n \cdot \frac{m_{\mathrm{mon}}}{z}+\frac{m_{\mathrm{end}}}{z}+m_{\mathrm{cat}}-m_{\mathrm{elec}}
\end{aligned}
$$

where $m_{\text {mon }}$ is the mass of the monomer unit, $n$ is the number of monomers, $m_{\text {end }}$ is the endgroup mass, $m_{\text {cat }}$ is the mass of the cation, and $m_{\text {elec }}$ is the electron mass. Eq 1 has to be extended with the term 


$$
x \cdot\left(\text { mass }{ }^{13} \mathrm{C} \text {-mass }{ }^{12} \mathrm{C}\right) / z
$$

if the selected oligomers contain $x{ }^{13} \mathrm{C}$ atoms. We have considered the masses of the cation and electron to be known exactly. Later in this paper we will describe a method that enables the determination of the identity of an unknown cation.

A more manageable way to determine the endgroup and monomer masses is by plotting the mass $m_{\text {meas }}$ [by multiplying $(\mathrm{m} / \mathrm{z})_{\text {meas }}^{z+}$ with the charge state] as a function of $n$ (eq 2) assuming that $z$ is known. In this equation the monomer mass and the sum of the endgroup and $z$ cation(s) follow immediately from the intercept and slope, respectively:

$$
m_{\text {meas }}=n \cdot m_{\text {mon }}+m_{\text {end }}+z \cdot m_{\text {cat }}-z \cdot m_{\text {elec }}
$$

In order to determine the degree of polymerization $n$ of a peak all possible combinations, $m_{\text {end }}=m_{0}+$ $n_{i} \cdot m_{\text {mon }}$ have to be considered. Here, $m_{0}$ is the smallest possible endgroup mass for $n_{i}=0,1,2, \ldots$ [6]. The calculated endgroup mass must be consistent with the information that is known about the polymer system (synthesis, etc).

Note that for unknown polymeric systems the mono isotopic peak might not be visible in the spectrum but only the isotopic peaks containing an unknown number of ${ }^{13} \mathrm{C}$ atoms. This results in a $m_{0}$ which is the sum of the endgroup and this unknown number of ${ }^{13} \mathrm{C}$ atoms. To determine the number of ${ }^{13} \mathrm{C}$ atoms the following procedure is followed. First, the monomer mass is the slope of eq 2, which is accurately determined (see further). Second, the elemental composition of the monomer is determined using the monomer mass. Third, by comparing the theoretical isotopic pattern of a given oligomer with the experimentally observed pattern, as done in Figure 2, the number of ${ }^{13} \mathrm{C}$ atoms a given peak contains is determined. Note that for relatively large monomer masses, e.g., >100 Da, various elemental compositions can correspond with the determined monomer mass. This requires additional information of the polymer from techniques like NMR.

For large $n$, the distance between the measured points and the intercept becomes large. As a result, small deviations in the slope will introduce a large error in the endgroup mass. This can easily be seen from the expression for the accuracy of the endgroup mass $\sigma_{\text {end }}^{2}$ that results from the propagation of errors in the extrapolation procedure

$$
\sigma_{\text {end }}^{2}=\sigma_{b}^{\prime 2}+\bar{n}^{2} \sigma_{a}^{\prime 2}=\frac{\sigma_{\text {data }}^{2}}{N}+\bar{n}^{2} \frac{\sigma_{\text {data }}^{2}}{\sum_{i=1}^{N} n_{i}^{\prime 2}}
$$

where $\sigma_{b}^{\prime 2}, \sigma_{a}^{\prime 2}$, and $\sigma_{\text {data }}^{2}$ are, respectively, the uncertainties in the center of gravity in the fit procedure, the slope and the mass measurements, $\bar{n}$ is the transformed average degree of polymerization as described in [6], $N$ is the number of data points, $n_{i}$ is an oligomer with degree of polymerization $i$ and $n_{i}^{\prime}=n_{i}-\bar{n}$. A more detailed description of eq 3 can be found in [6].

A method which is unaffected by small fluctuations in the slope has been evaluated by van Rooij et al. for MALDI FTICR-MS [6]. This method, "the averaging method," requires a known elemental composition of the monomer. The method does not involve an extrapolation procedure to $n=0$. By subtracting $n$ times the mass of the monomer from the mass of the singly charged ions formed by MALDI, the endgroup plus cation mass is obtained. This procedure is followed by an averaging of the results of all oligomers. For multiply charged ions, as formed by ESI, the same result is obtained by subtracting $n$ times the mass of the monomer from $m_{\text {meas. }}^{z+}$ The charge state follows from the spacing between the isotopic peaks. Hence isotopic resolution is absolutely necessary.

\section{Results of the Endgroup Determination Using the Linear Regression and Averaging Methods}

The results for the determination of the endgroup and monomer masses of PEG1000, PEG3000, and PEG6000 determined with linear regression ( $m_{\text {end,regression }}$ and $\left.m_{\text {mon,regression }}\right)$ and the averaging method ( $\left.m_{\text {end,average }}\right)$ are shown in Tables 1 and 2 . The endgroup masses have been calculated for all observed charge states separately. Only peaks with $\mathrm{S} / \mathrm{N}>3$ were used for the endgroup calculations.

The elemental composition of the monomers of PEG, PPG, and PTHF is confirmed with linear regression as $\mathrm{C}_{2} \mathrm{H}_{4} \mathrm{O}\left(m_{\text {mon,exact }}=44.0262 \mathrm{Da}, m_{\text {mon,regression }} \approx 44.025\right.$ $\mathrm{Da}), \mathrm{C}_{3} \mathrm{H}_{6} \mathrm{O}\left(m_{\text {mon,exact }}=58.0419 \mathrm{Da}, m_{\text {mon,regression }} \approx\right.$ $58.042 \mathrm{Da})$, and $\mathrm{C}_{4} \mathrm{H}_{8} \mathrm{O}\left(m_{\text {mon,exact }}=72.0575 \mathrm{Da}\right.$, $\left.m_{\text {mon,regression }} \approx 72.058 \mathrm{Da}\right)$, respectively. The mass accuracy resulting from linear regression is very high $\left(\Delta m_{\text {mon,regression }} \ll 0.1 \%\right)$ as can be seen from Tables 1 and 2 . Note that $m_{\text {mon,regression }}$ is determined for all charge states separately.

The endgroup mass $m_{\text {end,regression calculated with eq }}$ 2 includes the mass of the cation(s) as well (similar to the method used by van Rooij et al. [6]) and therefore increases with the charge state. When the mass of the cations and ${ }^{13} \mathrm{C}$ atoms is subtracted from the calculated endgroup, $m_{\text {end-cation,regression }}$ in Tables 1 and 2 , the elemental composition of the endgroups is confirmed as $-\mathrm{H}$ and $-\mathrm{OH}\left(m_{\text {end,exact }}=18.0106\right)$ for all polymers studied. This corresponds to what is known about the synthesis of polyoxyalkylene polymers. A comparison of the theoretical and the experimentally observed endgroup masses is described in more detail further in this paper.

The $\sigma_{\text {mon,regression }}$ and $\sigma_{\text {end,regression }}$ values are the uncertainties of, respectively, the monomer and endgroup mass as determined with the linear regression method. The $\sigma_{\text {end, average value is the uncertainty of }}$ 
Table 1. Calculated monomer and endgroup masses (Da) and accuracy for PEG1000 and PEG3000 of all observed charge states determined with the regression and averaging method. ${ }^{\text {a }}$ Only the monoisotopic peaks have been used for the determination of the exact masses

\begin{tabular}{|c|c|c|c|c|c|c|c|c|c|}
\hline \multirow{2}{*}{$\begin{array}{l}\text { Masses (Da) and } \\
\text { deviations }\end{array}$} & \multirow[b]{2}{*}{$z$} & \multicolumn{2}{|c|}{ PEG1000 } & \multicolumn{6}{|c|}{ PEG3000 } \\
\hline & & $1+$ & $2+$ & $2+$ & $3+$ & $4+$ & $5+$ & $6+$ & $7+$ \\
\hline$n(N)$ & & 10-26 (17) & 20-32 (13) & $58-80(22)$ & $59-92(34)$ & $60-92(33)$ & $60-93(32)$ & $63-94(28)$ & $72-89(16)$ \\
\hline$m_{\mathrm{mon}, \mathrm{r}}$ & & 44.0266 & 44.0267 & 44.0244 & 44.0255 & 44.0263 & 44.0264 & 44.0262 & 44.0261 \\
\hline$\Delta m_{\text {mon,regression }}$ & & 0.0004 & 0.0005 & -0.0018 & -0.0007 & 0.0001 & 0.0002 & $<0.0001$ & -0.0001 \\
\hline$\sigma_{\text {mon,regression }}$ & & 0.0006 & 0.0008 & 0.0020 & 0.0005 & 0.0006 & 0.0006 & 0.0003 & 0.0005 \\
\hline$m_{\text {end,regression }}$ & & 40.9963 & 63.9788 & 64.0979 & 87.0287 & 109.9672 & 132.9421 & 155.9484 & 178.9434 \\
\hline$m_{\text {end-cation,regression }}$ & & 18.0071 & 18.0003 & 18.1194 & 18.0610 & 18.0104 & 17.9960 & 18.0131 & 18.0189 \\
\hline$\Delta m_{\mathrm{end}, \text { regression }}$ & & -0.0035 & -0.0102 & 0.1089 & 0.0504 & -0.0002 & -0.0145 & 0.0025 & 0.0083 \\
\hline$\sigma_{\text {end,regression }}$ & & 0.0108 & 0.0197 & 0.1365 & 0.0399 & 0.0470 & 0.0487 & 0.0207 & 0.0429 \\
\hline$m_{\text {end,av }}$ & & 41.0031 & 63.9926 & 63.9768 & 86.9757 & 109.9699 & 132.9571 & 155.9453 & 178.9350 \\
\hline$m_{\text {end-cation, average }}$ & & 18.0139 & 18.0141 & 17.9983 & 18.0080 & 18.0130 & 18.0110 & 18.0106 & 18.0105 \\
\hline$\Delta m_{\text {end,average }}$ & & 0.0033 & 0.0036 & -0.0122 & -0.0025 & 0.0025 & 0.0005 & -0.0005 & -0.0001 \\
\hline$\sigma_{\text {end,average }}$ & & 0.0028 & 0.0028 & 0.0127 & 0.0051 & 0.0058 & 0.0060 & 0.0024 & 0.0028 \\
\hline$\sigma_{\text {data }}$ & & 0.0117 & 0.0101 & 0.0597 & 0.0300 & 0.0336 & 0.0340 & 0.0126 & 0.0110 \\
\hline
\end{tabular}

aThe $\Delta$ values denote the difference between the theoretical and measured masses. The $\sigma$ values are the corresponding standard deviations which

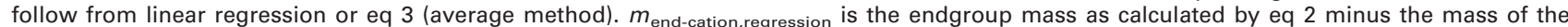
cation(s) and ${ }^{13} \mathrm{C}$ atoms. $m_{\text {end-cation, average }}$ is similar to $m_{\text {end-cation,regression }}$ but determined by the averaging method. $\sigma_{\text {data }}$ is considered for all charge states separately and is the maximum observed experimental mass error.

the endgroup mass as determined with the averaging method. Note that $\sigma_{\text {mon,average }}=0$ because the average method assumes the monomer mass to be known exactly. The determination of the accuracy in the experimental data $\left(\sigma_{\text {data }}\right)$ has been done for all charge states separately. We have taken the maximum observed experimental mass error in the measurements as $\sigma_{\text {data. }}$. The $\sigma_{\text {data }}$ value is considered for all charge states separately because the mass error increases with decreasing charge state (see further). Large fluctuations of $\sigma_{\text {data }}$ for the highest charge states have not been observed, e.g., for $3+-7+$ of PEG3000.

The range of oligomers $n$ and the corresponding number of datapoints $N$ observed for each charge state $z$ and used for the calculations are given in the tables. In most cases $N$ is determined by the range of oligomers $n$ observed in the spectrum. However, for some oligomers it was difficult to locate the peak because of peak overlap with other oligomers. Therefore, $N$ and the range of oligomers $n$ do not correspond in all cases.

The $m_{\text {end-cation,regression values of PEG1000 and }}$ PEG3000 are $~ 18.01$ Da which is less than $0.08 \%$ difference with the theoretical endgroup mass for $-\mathrm{OH}$ and $-\mathrm{H}$. For PEG6000 this value is smaller than $0.7 \%$. The $2+$ charge state of PEG3000 and $4+-5+$ charge states of PEG6000 are the only exceptions because of the decreasing mass accuracy at higher $\mathrm{m} / \mathrm{z}$ (see further). For PEG6000 and PPG3250 only the peaks containing 4 and $1^{13} \mathrm{C}$ isotopes were used for the endgroup and monomer calculations, respectively. This is necessary because the intensity of the monoisotopic peaks becomes too low.

The $\sigma_{\text {mon,regression }}$ and $\sigma_{\text {end,regression }}$ values are highest for the charge states which are observed in the $\mathrm{m} / \mathrm{z}$ range of $\sim 1300$ to 1800 . This corresponds to the $2+$ charge state for PEG3000, and the $4+$ and $5+$ charge states of
PEG6000. That the $\sigma_{\text {mon,regression }}$ and $\sigma_{\text {end,regression values }}$ are highest for these charge states can be seen in Figure 3 where the difference between exact and measured mass $\left(m_{\text {act }}-m_{\text {meas }}\right)$ is given for PEG3000 as a function of $m / z$. Only the $2+, 3+$, and $5+$ charge states have been plotted in Figure 3 to prevent an overlap of different charge states. The average mass error fluctuates around zero if $\mathrm{m} / \mathrm{z}$ is between 500 and 1200. An increase of the mass error is observed for $\mathrm{m} / \mathrm{z}$ values higher than 1200 . This increase in mass error at higher $\mathrm{m} / \mathrm{z}$ is attributed to the decreasing number of datapoints that constitutes a peak. Peaks of higher $\mathrm{m} / \mathrm{z}$ have a lower cyclotron frequency and are therefore reconstructed with less data acquisition points. This decrease in accuracy at higher $m / z$ results in higher $\sigma_{\text {mon,regression }}$ and $\sigma_{\text {end,regression }}$ values. Another consequence of the increasing mass error with $\mathrm{m} / \mathrm{z}$ is that the slope of the fitted line (eq 2) will decrease, which results in lower $m_{\text {mon,regression }}$ and higher $m_{\text {end,regression }}$ values. This can easily be seen from the tables where the $\Delta m_{\text {mon,regression }}$ value becomes more negative for lower charge states while $\Delta m_{\text {end,regression }}$ increases. In general, the best mass accuracy is achieved at the highest charge states.

The linear regression procedure requires an extrapolation from about $n=60$ to $n=0$ for PEG3000. Small deviations in the slope of the line will dramatically alter the extrapolated endgroup mass. This does not hold for the average method since this method does not require an extrapolation. For PEG1000, PPG1150, PPG3250, and PTHF2000 higher $\sigma_{\text {mon,regression }}$ and $\sigma_{\text {end,regression }}$ values at lower charge states, compared to higher charge states, are not observed. PEG1000 and PPG1150 are observed below $\mathrm{m} / \mathrm{z} 1300$ where the mass accuracy is relatively high. The lowest charge states $(2+$ and $3+)$ observed for PPG3250 contain at least twice as many datapoints as the higher charge states and increase the 

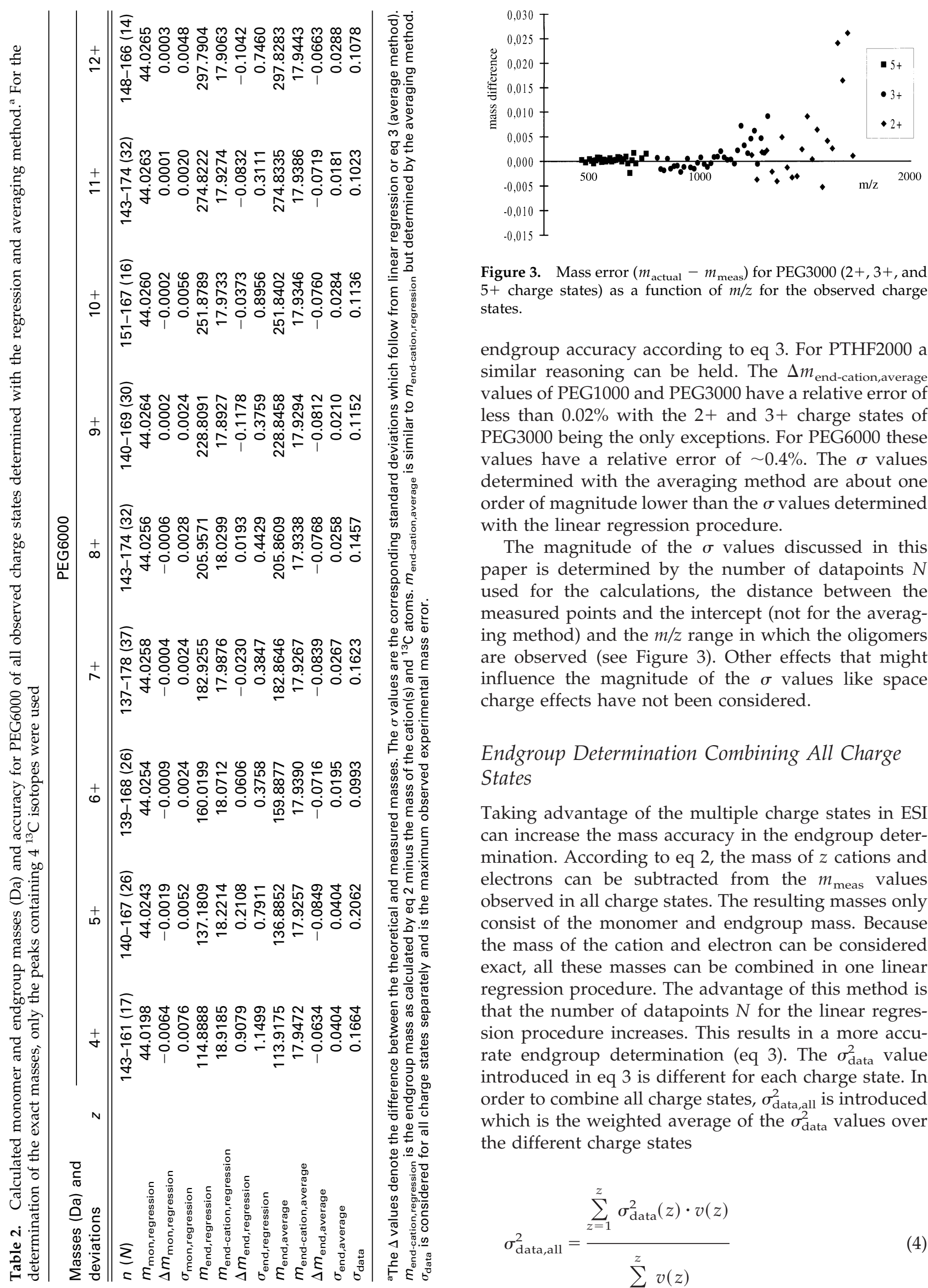

Figure 3. Mass error $\left(m_{\text {actual }}-m_{\text {meas }}\right)$ for PEG3000 $(2+, 3+$, and $5+$ charge states) as a function of $\mathrm{m} / \mathrm{z}$ for the observed charge states.

endgroup accuracy according to eq 3. For PTHF2000 a similar reasoning can be held. The $\Delta m_{\text {end-cation,average }}$ values of PEG1000 and PEG3000 have a relative error of less than $0.02 \%$ with the $2+$ and $3+$ charge states of PEG3000 being the only exceptions. For PEG6000 these values have a relative error of $\sim 0.4 \%$. The $\sigma$ values determined with the averaging method are about one order of magnitude lower than the $\sigma$ values determined with the linear regression procedure.

The magnitude of the $\sigma$ values discussed in this paper is determined by the number of datapoints $N$ used for the calculations, the distance between the measured points and the intercept (not for the averaging method) and the $\mathrm{m} / \mathrm{z}$ range in which the oligomers are observed (see Figure 3). Other effects that might influence the magnitude of the $\sigma$ values like space charge effects have not been considered.

\section{Endgroup Determination Combining All Charge States}

Taking advantage of the multiple charge states in ESI can increase the mass accuracy in the endgroup determination. According to eq 2, the mass of $z$ cations and electrons can be subtracted from the $m_{\text {meas }}$ values observed in all charge states. The resulting masses only consist of the monomer and endgroup mass. Because the mass of the cation and electron can be considered exact, all these masses can be combined in one linear regression procedure. The advantage of this method is that the number of datapoints $N$ for the linear regression procedure increases. This results in a more accurate endgroup determination (eq 3). The $\sigma_{\text {data }}^{2}$ value introduced in eq 3 is different for each charge state. In order to combine all charge states, $\sigma_{\text {data, all }}^{2}$ is introduced which is the weighted average of the $\sigma_{\text {data }}^{2}$ values over the different charge states

$$
\sigma_{\text {data, all }}^{2}=\frac{\sum_{z=1}^{z} \sigma_{\text {data }}^{2}(z) \cdot v(z)}{\sum_{z=1}^{z} v(z)}
$$




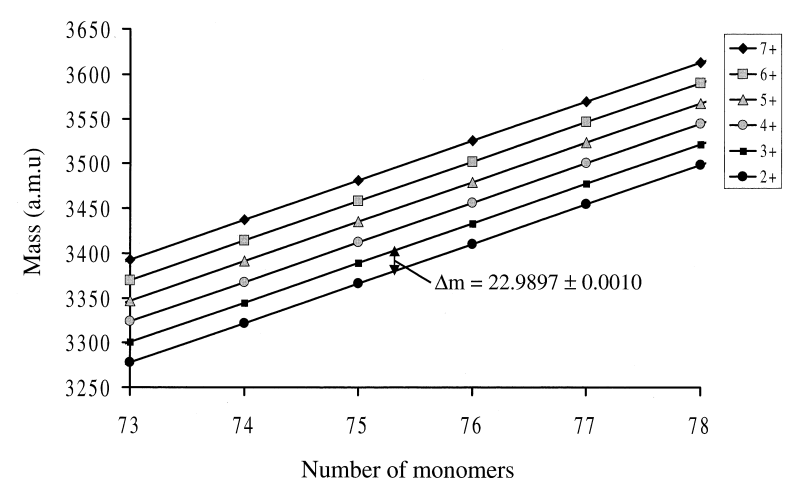

Figure 4. Determination of the mass of the cation for PEG3000. Plot of an enlarged mass scale of all observed charge states. The distance between two adjacent parallel lines determines the mass of the cation.

Where $\sigma_{\text {data }}^{2}(z)$ is the $\sigma_{\text {data }}^{2}$ value in charge state $z$ and $v(z)$ is the statistical degree of freedom of the number of datapoints $N$ observed in charge state $z$.

The charge carrying cation is sodium in all cases. This is confirmed by the addition of different alkali salts to the solution (spectra not shown). A major disadvantage of this method for cation identification is that additional measurements with other cations have to be carried out. A faster and less laborious confirmation is obtained in Figure 4 for PEG3000. In this figure the measured masses $\left(m_{\text {meas }}\right)$ in all charge states are plotted as a function of $n$. We have used the same $m_{\text {meas }}$ values as we have used for the endgroup determination. The vertical spacing between the different lines gives the mass of the cation. This can also be seen from eq 2 if $z$ is increased by one charge. For PEG3000 a cation mass of $22.989 \pm 0.001$ is determined from Figure 4 .

The results of the method that combines the data of all charge states are shown in Table 3. In general, the endgroup accuracy increases when all charge states are combined instead of considering them separately. This is explained as due to the increasing number of datapoints $N$ that is used in the linear regression procedure (eq 3). For PEG1000 a $\sigma_{\text {end,average }}$ of 0.0020 is observed after combining the two observed charge states. When considering the charge states separately, a 1.4 times higher value of 0.0028 is found. For PEG3000 a value of 0.0026 is observed which is on average 2.2 times higher than the $\sigma_{\text {end,average }}$ values of the separate charge states. For PEG6000 a factor of approximately 3 is observed. Similar results are obtained for PPG1150 and PPG3250.

\section{Comparison of the Endgroup Accuracy Determined with ESI and MALDI FTICR-MS}

The mass accuracies for PEG1000 and PEG4000 found by van Rooij et al. [6] with MALDI FTICR-MS are compared with the results for the PEGs discussed in this report (see summary of the MALDI results [6] in the last two columns of Table 3). The $\sigma$ values for PEG1000 determined with MALDI FTICR-MS are in good agreement with the values found in this report with ESI FTICR-MS when all charge states are considered separately. The $\sigma$ values of PEG4000 are of the same order of magnitude as those of PEG3000 and PEG6000. This demonstrates that the $\sigma$ values generated with ESI data are in good agreement with MALDI data. Note that the number of datapoints used with MALDI FTICR-MS in [6] is lower than the number of datapoints used in this paper with ESI FTICR-MS. This influences the $\sigma$ values. Therefore, the endgroup and monomer mass calculations have been done with a similar number of datapoints as used in [6] for several charge states of PEG3000 and PEG6000. As expected, the resulting $\sigma$ values are lower. However, the $\sigma$ values are still of the same order of magnitude as the results found with MALDI for PEG4000.

The $\sigma$ values of PEG6000 have improved by a factor of about 2 compared to those of PEG4000 when all charge states are combined in one linear regression procedure (fourth column of Table 3). This is in spite of the fact that the molecular weight of PEG6000 is larger than that of PEG4000. Large oligomers can be measured more accurately using ESI compared to MALDI because

Table 3. Calculated monomer and endgroup masses (Da) and accuracy for PEG1000, PEG3000, PEG6000, PPG1150, PPG3250, and PTHF2000 combining all charge states. The values of PEG1000 and PEG4000 displayed in the last two columns are obtained with MALDI FT-ICRMS from [6] (mass of cation and ${ }^{13} \mathrm{C}$ subtracted)

\begin{tabular}{|c|c|c|c|c|c|c|c|c|}
\hline \multirow{2}{*}{$\begin{array}{l}\text { Masses (Da) and } \\
\text { deviations }\end{array}$} & \multirow[b]{2}{*}{ PEG1000 } & \multirow[b]{2}{*}{ PEG3000 } & \multirow{2}{*}{$\begin{array}{l}\text { PEG6000 } \\
\left(+4{ }^{13} \mathrm{C}\right) \\
\end{array}$} & \multirow[b]{2}{*}{ PPG1150 } & \multirow{2}{*}{$\begin{array}{l}\text { PPG3250 } \\
\left(+1{ }^{13} \mathrm{C}\right) \\
\end{array}$} & \multirow[b]{2}{*}{ PTHF2000 } & PEG1000 & PEG4000 \\
\hline & & & & & & & \multicolumn{2}{|c|}{ values from [6] } \\
\hline$n(N)$ & $10-32(30)$ & $58-94(165)$ & $137-178(230)$ & $6-25(29)$ & $16-72(134)$ & $4-29(41)$ & $13-30(18)$ & 85-102 (18) \\
\hline$m_{\text {mon,regression }}$ & 44.0265 & 44.0261 & 44.0256 & 58.0421 & 58.0419 & 72.0577 & 44.0260 & 44.0285 \\
\hline$\Delta m_{\text {mon, regression }}$ & 0.0002 & -0.0001 & -0.0006 & 0.0002 & $<0.0001$ & 0.0002 & -0.0002 & 0.0023 \\
\hline$\sigma_{\text {mon,regression }}$ & 0.0003 & 0.0003 & 0.0010 & 0.0003 & 0.0001 & 0.0003 & 0.0004 & 0.0025 \\
\hline$m_{\text {end-cation, regression }}$ & 18.0087 & 18.0164 & 18.0273 & 18.0085 & 18.0088 & 18.0117 & 18.0120 & 17.7941 \\
\hline$\Delta m_{\text {end,regression }}$ & -0.0019 & 0.0059 & 0.0167 & -0.0021 & -0.0018 & 0.0011 & 0.0014 & -0.2200 \\
\hline$\sigma_{\text {end,regression }}$ & 0.0076 & 0.0217 & 0.1631 & 0.0046 & 0.0058 & 0.0053 & 0.0080 & 0.2400 \\
\hline$m_{\text {end-cation, average }}$ & 18.0140 & 18.0089 & 17.9338 & 18.0117 & 18.0112 & 18.0153 & 18.0079 & 18.0081 \\
\hline$\Delta m_{\text {end, average }}$ & 0.0034 & -0.0017 & -0.0768 & 0.0012 & 0.0006 & 0.0047 & -0.0027 & -0.0030 \\
\hline$\sigma_{\text {end,average }}$ & 0.0020 & 0.0026 & 0.0093 & 0.0014 & 0.0017 & 0.0019 & 0.0019 & 0.0140 \\
\hline$\sigma_{\text {data,all }}$ & 0.0111 & 0.0339 & 0.1413 & 0.0074 & 0.0193 & 0.0124 & 0.0080 & 0.0600 \\
\hline
\end{tabular}


ESI yield lower $m / z$ values which can be measured more accurately using FTMS.

The largest oligomer observed with MALDI has a degree of polymerisation of 105. For ESI an oligomer with 178 monomeric units (PEG6000) has been observed on the same FTMS. This demonstrates clearly that when ESI is used instead of MALDI the mass range is extended by almost a factor of 2. Polyethylene glycol samples with molecular weights of up to $20,000 \mathrm{Da}$ have also been measured successfully using ESI. Accurate endgroup determinations for polyethylene glycol samples with a molecular weight larger than $20,000 \mathrm{Da}$ require a mass resolving power only provided by a high field FTMS.

\section{Conclusions}

Two methods, a linear regression and averaging method, have been developed and evaluated for the accurate analysis of the monomer and endgroup composition of synthetic polymers using ESI FTICR-MS. All charge states of a polymer, observed with ESI, are combined in one linear regression or averaging procedure, in order to increase the accuracy of the endgroup determination. The charge states can be combined under condition that the mass of the cation is known. By plotting $(\mathrm{m} / \mathrm{z})^{z+}$ multiplied by the charge state as a function of the degree of polymerization the identity of the cation is easily determined. This procedure prevents multiple experiments in which different cations have to be added to the polymer sample.

The endgroup accuracy determined from a single charge state with ESI FTICR-MS is of the same order of magnitude as determined by MALDI FTICR-MS on the same instrument. However, when all charge states measured with ESI are combined in one procedure, the endgroup accuracy increases up to threefold. This demonstrates that more accurate monomer and endgroup masses are obtained using ESI instead of MALDI. ESI exceeds MALDI in the study of larger polymeric systems due to the multiple charging nature of ESI. Large polymers have a lower $\mathrm{m} / \mathrm{z}$ value, which are measured with a higher accuracy in the FTMS.

\section{Acknowledgments}

The authors gratefully acknowledge A. Vijftigschild, I. Stavenuiter, A. de Snaijer, and S. Wouda for their technical assistance in the various stages of the experiment. We like to thank Ir. G. J. van Rooij, Dr. M. W. F. Nielen from Akzo Nobel Chemicals Research Arnhem, and Dr. C. G. de Koster from DSM Research for useful discussions. Jon Doye and Tina Weeding are acknowledged for reviewing this manuscript. This work is part of the research program nr. 28 "Mass Spectrometry of Macromolecular systems" of the "Stichting voor Fundamenteel Onderzoek der Materie (FOM)" a subsidiary of the "Nederlandse organisatie voor Wetenschappelijke Onderzoek (NWO)." This project is financially supported by FOM, DSM Research (Geleen, the Netherlands), and
Akzo Nobel Chemicals Research Arnhem (Arnhem, the Netherlands).

\section{References}

1. Montaudo, G.; Montaudo, M. S.; Puglisi, C.; Samperi, F. Rapid Commun. Mass Spectrom. 1995, 9, 453-460.

2. Montaudo, G.; Garozzo, D.; Montaudo, M. S.; Puglisi, C.; Samperi, F. Macromolecules 1995, 28, 7983-7989.

3. Nielen, M. W. F.; Malucha, S. Rapid Commun. Mass Spectrom. 1997, 11, 1194-1204.

4. Easterling, M. L.; Mize, T. H.; Amster, I. J. Int. J. Mass Spectrom. Ion Processes 1997, 169/170, 387-400.

5. McLafferty, F. W. Acc. Chem. Res. 1994, 27, 379-386.

6. van Rooij, G. J.; Duursma, M. C.; Heeren, R. M. A.; Boon, J. J.; de Koster, C. G. J. Am. Soc. Mass Spectrom. 1996, 7, 449-457.

7. Alber, G. M.; Marshall, A. G.; Hill, N. C.; Schweikhard, L.; Ricca, T. Rev. Sci. Instrum. 1993, 64, 1845-1852.

8. Dey, M.; Castoro, J. A.; Wilkins, C. L. Anal. Chem. 1995, 67, 1575-1579.

9. O'Connor, P. B.; Duursma, M. C.; van Rooij, G. J.; Heeren, R. M. A.; Boon, J. J. Anal. Chem. 1997, 69, 2751-2755.

10. Prokai, L.; Simonsick, W. J., Jr. Rapid Commun. Mass Spectrom. 1993, 7, 853-856.

11. Nielen, M. W. F. Rapid Commun. Mass Spectrom. 1996, 10, $1652-1660$

12. Reinhold, M.; Meier, R. J.; de Koster, C. G. Rapid Commun. Mass Spectrom. 1998, 12, 1962-1966.

13. Wong, S. F.; Meng, C. K.; Fenn, J. B. J. Phys. Chem. 1988, 92, 546-550.

14. Jasieczek, C. B.; Buzy, A.; Haddleton, D. M.; Jennings, K. R. Rapid Commun. Mass Spectrom. 1996, 10, 509-514.

15. McEwen, C. N.; Simonsick, W. J., Jr.; Larsen, B. S.; Ute, K.; Hatada, K. J. Am. Soc. Mass Spectrom. 1995, 6, 906-911.

16. Hofstadler, S. A.; Beu, S. C.; Laude, D. A., Jr. Anal. Chem. 1993, 65, 312-316.

17. Gard, E. E.; Green, M. K.; Warren, H.; Camara, E. J. O.; He, F.; Penn, S. G.; Lebrilla, C. B. Int. J. Mass Spectrom. Ion Processes 1996, 157/158, 115-127.

18. O'Connor, P. B.; McLafferty, F. W. J. Am. Chem. Soc. 1995, 117, 12826-12831.

19. Schmidt, E. G.; Karpinski, M. L.; Bolanos, B. J.; Harper, C. J.; Weil, D. A.; Laude, D. A. Proceedings of the 45th ASMS Conference on Mass Spectrometry and Allied Topics; Palm Springs, 1997; p 881.

20. Mize, T. H.; Pitsenberger, C. C.; Easterling, M. L.; Danis, P.; Amster, I. J. Proceedings of the 45th ASMS Conference on Mass Spectrometry and Allied Topics; Palm Springs, 1997; p 542.

21. Pastor, S. J.; Wilkins, C. L. Proceedings of the 45th ASMS Conference on Mass Spectrometry and Allied Topics; Palm Springs, 1997; p 537.

22. van der Hage, E. R. E.; Duursma, M. C.; Heeren, R. M. A.; Boon, J. J.; Nielen, M. W. F.; Weber, A. J. M.; de Koster, C. G.; de Vries, N. K. Macromolecules 1997, 30, 4302-4309.

23. de Koster, C. G.; Duursma, M. C.; van Rooij, G. J.; Heeren, R. M. A.; Boon, J. J. Rapid Commun. Mass Spectrom. 1995, 9, 957-962.

24. Easterling, M. L.; Amster, I. J.; van Rooij, G. J.; Heeren, R. M. A. J. Am. Soc. Mass Spectrom., submitted.

25. Caravatti, P.; Allemann, M. Org. Mass Spectrom. 1991, 26, 514-518.

26. Heeren, R. M. A.; de Koster, C. G.; Boon, J. J. Anal. Chem. 1995, $67,3965-3970$.

27. Heeren, R. M. A.; Boon, J. J. Int. J. Mass Spectrom. Ion Processes 1996, 157/158, 391-403.

28. Nohmi, T.; Fenn, J. B. J. Am. Chem. Soc. 1992, 114, 3241-3246. 